Article

\title{
Fast, Nondestructive, and Broadband Dielectric Characterization for Polymer Sheets
}

\author{
Hsin-Yu Yao®, Dan-Ru Hsiao and Tsun-Hsu Chang *(D) \\ Department of Physics, National Tsing Hua University, 101 Section 2 Kuang Fu Road, Hsinchu 300, Taiwan; \\ s5te633v@hotmail.com (H.-Y.Y.); goo@gapp.nthu.edu.tw (D.-R.H.) \\ * Correspondence: thschang@phys.nthu.edu.tw; Tel.: +886-(03)-5742-978
}

Received: 30 July 2020; Accepted: 18 August 2020; Published: 21 August 2020

check for updates

\begin{abstract}
We propose a compact nearfield scheme for fast and broadband dielectric characterization in the microwave region. An open-type circular probe operated in the high-purity $\mathrm{TE}_{01}$ mode was developed, showing a strongly confined fringing field at the open end. This fringing field directly probed the freestanding sheet sample, and the overall reflection was measured. Without sample-loading processes, both of the system assembling time and the risk of sample damage can be significantly reduced. In addition, the nearfield measurement substantially simplifies the calibration and the retrieval theory, facilitating the development of easy-to-integrate and easy-to-calibrate dielectric characterization technique. The dielectric properties of more than ten polymers were characterized from $30 \mathrm{GHz}$ to $40 \mathrm{GHz}$. We believe that this work fulfills the requirement of the fast diagnostic in the industrial manufactures and also provides valuable high-frequency dielectric information for the designs of $5 \mathrm{G}$ devices.
\end{abstract}

Keywords: polymer dielectric characterization; nearfield measurement; mode converter; transmission/reflection method

\section{Introduction}

With the advent of the 5th generation mobile networks (5G), numerous high-frequency circuits, such as power amplifiers [1,2], flexible antennas [3], and low-pass filters [4], are required for connecting billions of mobile devices and sharing a massive amount of data. To improve the performance and the integration of these microdevices, reducing the signal loss within the circuits and inhabiting the leakage current in the transistors become important tasks. For designing and optimizing, it is essential to investigate the broadband dielectric properties of the circuit-board materials. On the other hand, the nanocomposite field has grown quickly in recent years [5-8]. By doping dielectric/magnetic nanoparticles into polymer matrices, the electromagnetic properties of the composites can be precisely controlled, realizing many terahertz $(\mathrm{THz})$ and optical devices such as multilayer anti-reflection coatings [9], and all-dielectric waveguides [10]. To understand the electric functionality of the nanocomposites, fast and broadband dielectric characterization techniques at the microwave to the terahertz regimes are also required. Over the past decades, various methods have been developed to meet this goal from $1 \mathrm{GHz}$ to $1 \mathrm{THz}$ [11-20].

The conventional cavity methods, utilizing the field enhancement of the resonant nature, are able to characterize the materials with various permittivity $[12,13]$. However, those methods are very narrowband owing to the strict resonant conditions. For broadband characterizations, non-resonant techniques that measure the scattering parameters (transmission/reflection) in waveguides were invented [11,14-17]. Since the samples are enclosed by the waveguides and all experimental components (e.g., adapters and widows) can be well-calibrated, accurate and stable broadband results are expected. Nevertheless, sample preparation for these methods is usually 
difficult [14-16]. On the one hand, machining samples to meet the waveguide geometry is costly and might lead to sample damage. On the other hand, it is time-consuming to integrate the measurement setup due to the processes of sample loading, position/orientation adjustments, and the avoidance of the contact gaps [17].

The quasi-optical technique in the microwave region [18-20], and the time-domain spectroscopy in the terahertz region [21-23], can overcome these challenges. It composes of a set of antennas (source and receiver) to apply the far-field measurement in free space [18-20]. Unfortunately, the beam diffraction or the employment of multiple focusing devices severely complicate the experimental setups and the calibration procedures. In short, there is an urgent need to develop a compact, easy-to-integrate, and easy-to-calibrate dielectric characterization system.

In this work, we propose an open-ended circular-waveguide probe for fast and broadband dielectric characterization. The probe operated in the circular $\mathrm{TE}_{01}$ mode $[24,25]$ is directly attached to a freestanding sheet sample. Since there is no need to load the sample, the sample preparation time, the system assembling time, and the risk of sample damage can be greatly reduced as compared to References [11-17]. On the other hand, the $\mathrm{TE}_{01}$ mode exhibits purely azimuthal surface current, implying very weak diffraction as it radiates from the waveguide to the sample. This unique feature facilitates the nearfield measurement, making the calibration much easier than References [18-20]. We developed a semi-analytical model with a reliable single-mode approximation to simplify the dielectric retrieval process. More than ten polymer sheets were tested, and the result shows good agreement with the full-wave simulation results as well as the previously reported data [26-36].

\section{Scheme for the Fast Dielectric Characterization}

Simplifying the sample-loading process (necessary for References [11-17]) is a major challenge for the fast dielectric characterization. Inspired by the quasi-optical techniques [18-20], we propose an open-ended configuration delineated in Figure 1a, in which the end of a circular waveguide (Region I) directly probes the front surface of the sample at Region II. The sample with a thickness of $d$ is freestanding in open space, and its rear side is attached to a metal plate for enhancing the total reflection. To avoid the beam diffraction and the derived complexity in the calibrations (suffered by the far-field measurements [18-20]), properly choosing the operating waveguide mode is necessary.

Considering the $\mathrm{TE}_{01}$ mode in an empty circular $d R_{w}$ waveguide with a radius of $R_{w}$, its electromagnetic (EM) field components are [37],

$$
\begin{gathered}
E_{\phi}=\frac{-i k_{0}}{k_{c}} E_{0} J_{0}^{\prime}\left(k_{c} \rho\right) e^{i k_{z 0} z-i \omega t} \\
H_{\rho}=\frac{i k_{z 0}}{k_{c}} H_{0} J_{0}^{\prime}\left(k_{c} \rho\right) e^{i k_{z 0} z-i \omega t} \\
H_{z}=H_{0} J_{0}\left(k_{c} \rho\right) e^{i k_{z} z-i \omega t}
\end{gathered}
$$

where $\omega$ is the angular frequency of the EM wave, and $E_{0}\left(H_{0}\right)$ represents the electric-field (magnitude-field) amplitude. $k_{0}=\omega / c, k_{c}=0.382 / R_{w}$, and $k_{\mathrm{z} 0}^{2}=k_{0}^{2}-k_{c}^{2}$, respectively, describe the free-space wavenumber, the cutoff wavenumber, and the propagation constant, where $c$ is the speed of light in vacuum. The notation $J_{0}^{\prime}$ refers to the derivative of $J_{0}$ (first-kind Bessel function). All the other field components (not included in Equation (1)) are equal to zero.

The electric field of the $\mathrm{TE}_{01}$ mode is purely azimuthal, driving an azimuthal surface current on the waveguide wall $(\propto \hat{\rho} \times \vec{H})$. Such azimuthal surface current is insensitive to the radial geometric perturbations [37], such as the slotted waveguide structures [38], and the open-ended junction proposed here. This special feature guarantees that the fringing field in Region II can still preserve the same field pattern and the dispersion of the $\mathrm{TE}_{01}$ mode, propagating over a reasonably long distance in the sample. In other words, the beam diffraction can be significantly suppressed.

For verification, we used HFSS (High-Frequency Structure Simulator, ANSYS, Canonsburg, RA, USA) to simulate the steady-state field distributions (with $R_{w}=6.02 \mathrm{~mm}, R_{s}=15 \mathrm{~mm}$, and $d=2 \mathrm{~mm}$ ). 
The side edges of Region II (at $\rho=R_{s}$ ) were set as the radiation boundaries to save the computation time. The result of the $\mathrm{TE}_{01}$ incidence is demonstrated in Figure $1 \mathrm{a}$, while that for the $\mathrm{TE}_{11}$ incidence (i.e., the fundamental circular waveguide mode) is shown in Figure $1 \mathrm{~b}$ for comparison. The fringing field of the $\mathrm{TE}_{01}\left(\mathrm{TE}_{11}\right.$ ) mode in Region II is highly (weakly) confined, indicating very weak (strong) diffraction. The radiation losses (defined by $1-|R|^{2} ; R$ is the total field reflection coefficient) of the first five lower-order modes $\left(\mathrm{TE}_{11}, \mathrm{TM}_{01}, \mathrm{TE}_{21}, \mathrm{TM}_{11}\right.$, and $\left.\mathrm{TE}_{01}\right)$ are shown in Figure 1c. The loss of the $\mathrm{TE}_{01}$ mode is negligible, corresponding to the highly confined fringing field, as shown in Figure 1a. On the contrary, the radiation losses of the other modes are all higher than $5 \%$, owing to their highly diffracted feature (e.g., Figure $1 \mathrm{~b}$ for $\mathrm{TE}_{11}$ ). The $\mathrm{TE}_{01}$ radiation losses under three different thicknesses $(d=1,2$, and $3 \mathrm{~mm})$ are illustrated in Figure 1d. As shown, the loss remains low with the increase of the free-propagating length. Consequently, the $\mathrm{TE}_{01}$ mode is the best candidate for nearfield reflection measurement. This scheme combines the advantages of the waveguide systems [11-17] (highly confined field and easy-to-calibrate), and the advantage of the quasi-optical systems [18-20] (simple setup and easy-to-integrate), presenting the possibility for fast, efficient, and broadband dielectric characterization.
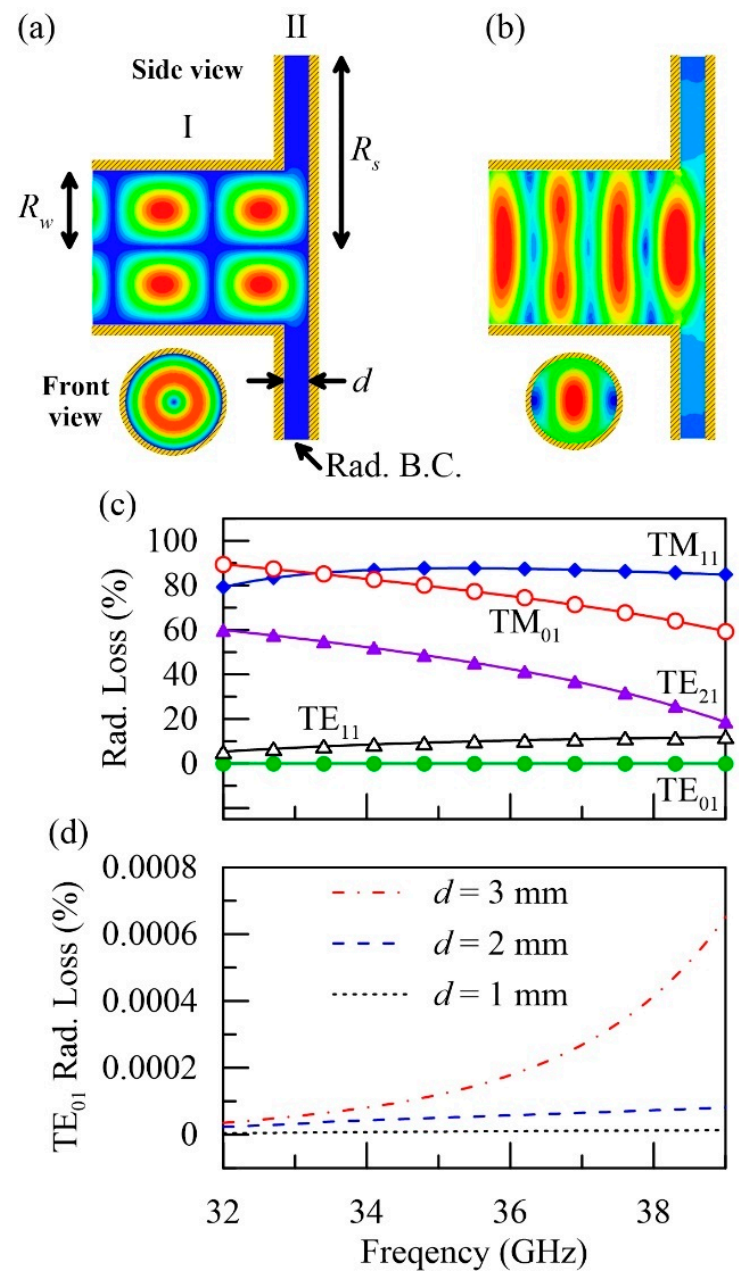

Figure 1. Fringing field patterns of the open-ended circular waveguide probe operated at (a) $\mathrm{TE}_{01}$ mode and (b) $\mathrm{TE}_{11}$ mode. (c) Radiation loss versus frequency of the first five circular waveguide modes. (d) Radiation loss versus frequency of the $\mathrm{TE}_{01}$ mode under the three different sample (air) thicknesses.

\section{Theoretical Model for the Complex Permittivity Retrieval}

A concise theory is developed for retrieving sample's complex permittivity from the measured reflection coefficient. When the $\mathrm{TE}_{01}$ mode impinges on the open end $(z=0)$, it will be partially 
reflected (as the ballistic reflection) and partially radiated into the sample. The radiation propagates over the sample and then is reflected by the metal plate $(z=d)$. The reflected signal subsequently bounces back and forth inside the sample, forming a sequence of reflection echoes. The steady-state total reflection is the superposition of the ballistic reflection and the all following echoes.

In general, the complexity of the coupling between a waveguide mode and the open-space diffracted modes (i.e., the plane waves propagating toward different directions) is very high. By taking advantage of the highly confined $\mathrm{TE}_{01}$ fringing field in open space, it is possible to simplify the model. The original scheme is illustrated in Figure 2a, in which the sample cross-section is assumed to be much larger than the wavelength $\left(R_{S}>>\lambda\right)$. Since the fringing field can never reach the sample edge (highlighted by the red dashes in Figure 2a), it is reasonable to replace the radiation boundaries by the metallic boundaries. The original "open system" thus becomes a "closed system" (Figure 2b). However, as the waveguide geometry in Figure $2 \mathrm{~b}$ is discontinuous at $z=0$, multiple higher-order $\mathrm{TE}_{0 m}$ modes must be excited at the junction, jointly with the $\mathrm{TE}_{01}$ mode to satisfy the boundary conditions. This process is known as the modal effect [37].
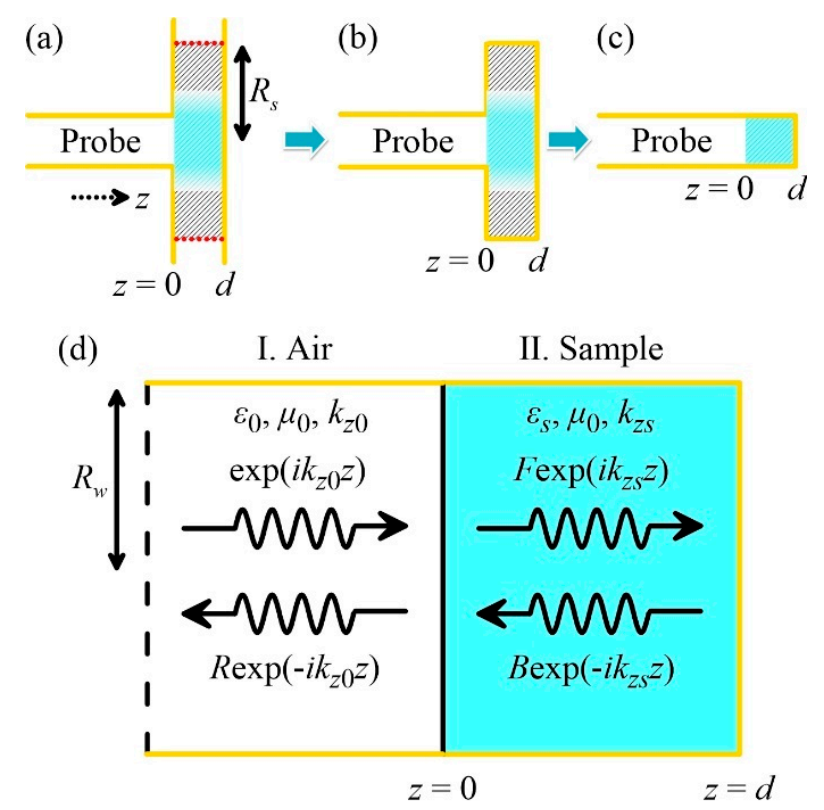

Figure 2. Simplification procedure of the theoretical retrieval model. (a) Original open-ended scheme. Red dotted lines indicate the radiation boundaries on the side edges. (b) First-step simplification: a closed system with the geometric change in the waveguide radius. The radiation boundaries in (a) are replaced by the metals. (c) Second-step simplification: a closed and uniform waveguide system. (d) Magnified diagram of (c) with the details for the single-mode approximation.

Dealing with the modal analysis $[10,17,39]$ is not the main focus of this work; instead, we further simplify the model according to Figure $2 \mathrm{c}$. Owing to the strong field confinement of the $\mathrm{TE}_{01}$ radiation (as demonstrated in Figure 1a), Figure $2 \mathrm{~b}$ is approximately equivalent to a uniform and closed-ended waveguide with the under-test sample locating at its terminal. We can therefore adopt the single-mode approximation (with only $\mathrm{TE}_{01}$ ) to solve the overall reflection. It is worth emphasizing that this single-mode approximation is not valid for other operating modes that exhibit highly diffracted fringing fields in open space (e.g., the lower-order modes listed in Figure 1c).

Based on this simplified configuration, the steady-state fields in each region are shown in Figure 2d. The transverse field components in the empty waveguide (Region I) can be expressed as

$$
\begin{gathered}
E_{\phi}^{\mathrm{I}}=\frac{-i \omega \mu_{0}}{k_{c}} J_{0}^{\prime}\left(k_{c} \rho\right)\left(e^{i k_{z 0} z}+R e^{-i k_{z 0} z}\right) e^{-i \omega t} \\
H_{\rho}^{\mathrm{I}}=\frac{i k_{z 0} 0}{k_{c}} J_{0}^{\prime}\left(k_{c} \rho\right)\left(e^{i k_{z 0} z}-R e^{-i k_{z 0} z}\right) e^{-i \omega t}
\end{gathered}
$$


where $R$ represents the overall field reflection coefficient. The transverse field components in the sample region (with permittivity $\varepsilon_{s}$ and permeability $\mu_{0}$ ) are

$$
\begin{gathered}
E_{\phi}^{\mathrm{II}}=\frac{-i \omega \mu_{0}}{k_{c}} J_{0}^{\prime}\left(k_{c} \rho\right)\left(F e^{i k_{z S} z}+B e^{-i k_{z S} z}\right) e^{-i \omega t} \\
H_{\rho}^{\mathrm{II}}=\frac{i k_{z s}}{k_{c}} J_{0}^{\prime}\left(k_{c} \rho\right)\left(F e^{i k_{z s} z}-B e^{-i k_{z s} z}\right) e^{-i \omega t}
\end{gathered}
$$

in which $F(B)$ denotes the forward-wave (backward-wave) coefficient and $k_{z s}^{2}=\mu_{0} \varepsilon_{s} \omega^{2}-k_{c}^{2}$. Applying the boundary conditions at $z=0$ that require $\left.E_{\phi}^{\mathrm{I}}\right|_{z=0}=\left.E_{\phi}^{\mathrm{II}}\right|_{z=0}$ and $\left.H_{\rho}^{\mathrm{I}}\right|_{z=0}=\left.H_{\rho}^{\mathrm{II}}\right|_{z=0}$ and the boundary condition at $z=L$ that forces $E_{\phi}^{\mathrm{II}} \mid z=L=0$ due to the metallic reflector, we obtain

$$
R=\frac{-\left(k_{z 0}-k_{z s}\right) e^{-2 i k_{z s} d}+\left(k_{z 0}+k_{z s}\right)}{-\left(k_{z 0}+k_{z s}\right) e^{-2 i k_{z s} d}+\left(k_{z 0}-k_{z s}\right)}
$$

Although Equation (4) resembles the solution of the two-section configuration with one end closed (Figure 2c), it serves as an approximated solution for the present "open scheme" as long as we operate with the circular $\mathrm{TE}_{01}$ modes. Note that Equation (4) is a transcendental equation; numerical root searching is thus required to retrieve the sample's permittivity $\varepsilon_{s}$ embedded in $k_{z s}$.

\section{Experimental Setup and Comparison with HFSS Simulation}

The photograph of the experimental setup is demonstrated in Figure 3a. Part I is the performance network analyzer (PNA, Agilent E8363B, Agilent Technologies, Santa Clara, CA, USA), which was connected with a $2.4 \mathrm{~mm}$ coaxial cable (part II). This cable was attached to an adapter (part III), converting the TEM signal to the $\mathrm{TE}_{10}$ mode in the $\mathrm{K}_{\mathrm{a}}$-band rectangular waveguide and vice versa. For fast dielectric characterization, exciting a high-purity $\mathrm{TE}_{01}$ mode in a circular waveguide is essential. Based on our previous works [24,25], a typical Y-type $\mathrm{TE}_{01}$ mode converter was designed, fabricated, and connected at part IV, severing as the open-ended circular probe. It comprises two-stage power-dividing junctions made of branched $\mathrm{K}_{\mathrm{a}}$-band rectangular waveguides (part $\mathrm{A}$ in Figure $3 \mathrm{~b}$ ), followed by a mode-converting section made of circular waveguide (part B in Figure 3b). According to the HFSS simulation, the purity of the $\mathrm{TE}_{01}$ mode achieves more than $99 \%$ ranging from $32 \mathrm{GHz}$ to $39 \mathrm{GHz}[10,25]$ (see Supplementary Materials S1 for its detail characteristics). A sheet sample (part V) is sandwiched between the $\mathrm{TE}_{01}$ mode converter (part IV) and a metal plate (part VI). The cross-sectional areas of all the samples are $80 \mathrm{~mm} \times 80 \mathrm{~mm}$, much larger than the probe size with $R_{w}=6.02 \mathrm{~mm}$. The sample thicknesses range between $1 \mathrm{~mm}$ and $2 \mathrm{~mm}$. Notice that the reference plane is calibrated to the interface between parts IV and V, as indicated by the red dashed line in Figure 3a (see Supplementary Materials S2 for the detail calibration procedure). The overall reflection was recorded by the PNA and analyzed with Equation (4) to retrieve the complex permittivity.

For verification, we used HFSS to simulate all the above procedures, including the calibration of the mode converter and the permittivity retrieval of an artificial under-test material. The relative permittivity $\left(\varepsilon_{r}\right)$ of the under-test sample is set as 4 , and its loss tangent $(\tan \delta)$ is set as 0.02 over the whole spectral window. The sample thickness is $1 \mathrm{~mm}$. With the scattering data of the mode converter (see Supplementary Materials S2), we can extract the complex permittivity of the sample under test based on Equation (4). The result is plotted in Figure $3 \mathrm{c}$ to compare with the default values. The retrieved $\varepsilon_{r} \sim 4.009$ shows excellent agreement with the default with an error less than $0.5 \%$ over the whole spectral window. On the other hand, the retrieved $\tan \delta \sim 0.022$ is slightly larger than the default because of the open boundaries of the freestanding sample; however, the accuracy is still acceptable. To further improve the accuracy of $\tan \delta$, the modal analysis is required for the less-simplified configuration shown in Figure $2 b$, i.e., the closed waveguide system with a sharp radius change. 

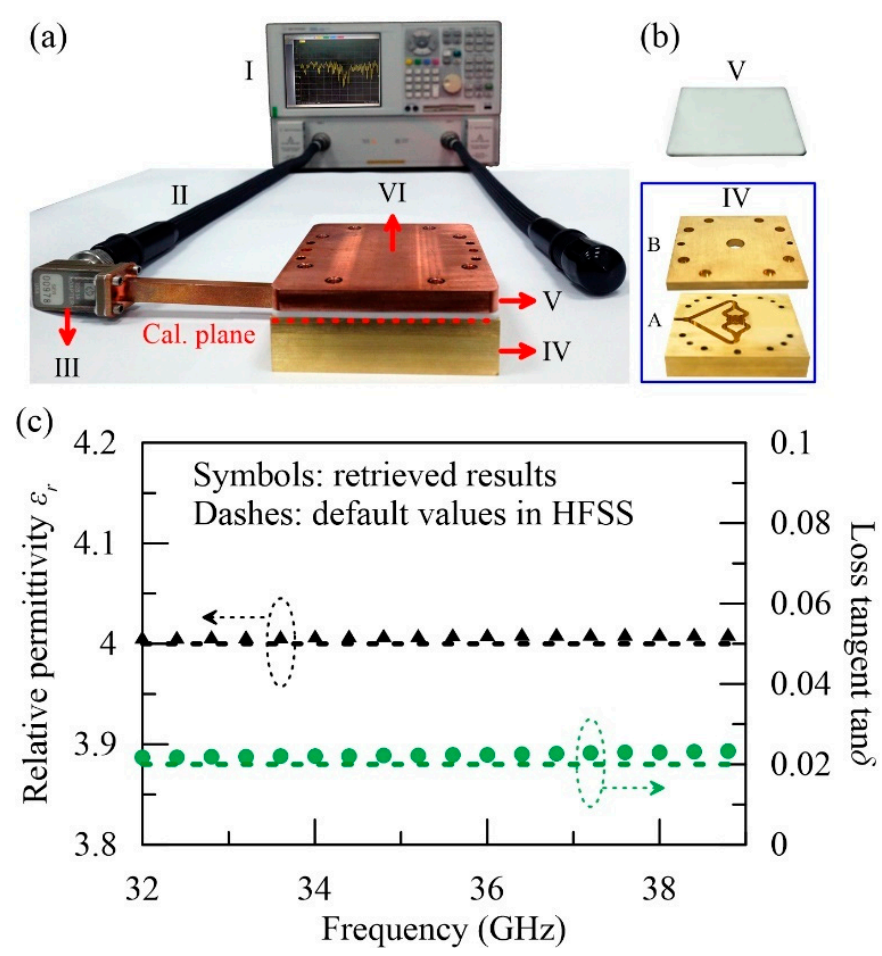

Figure 3. (a) Experimental setup. Part I: performance network analyzer. Part II: $2.4 \mathrm{~mm}$ flexible coaxial cable. Part III: rectangular $\mathrm{TE}_{10}$ mode converter. Part IV: home-made circular $\mathrm{TE}_{01}$ mode converter, composed of a power-dividing junction (component $\mathrm{A}$ in (b)) and a mode-converting tube (component B in (b)). Part V: sample under test. Part VI: back metal plate. (c) Retrieved results obtained by HFSS simulation. Black triangles (green circles): retrieved $\varepsilon_{r}(\tan \delta)$ based on the theory proposed in Section 3. The corresponding default values set in HFSS are plotted in dashed curves for comparison.

\section{Results and Discussions}

In the experiment, we characterized eleven polymers in total, including three common plastics (polyethylene (PE), polypropylene (PP), and polymethylmethacrylate (PMMA)), one heat/chemical-resisting plastic (polytetrafluoroethylene (PTFE)), one engineering plastic (polycarbonate (PC)), two piezoelectric materials (polyvinyl chloride (PVC) and polyvinylidene difluoride (PVDF)), and four circuit boards (FR4). Two of the FR4 boards are yellow (FR4_Y $\mathrm{Y}_{\mathrm{a}}$ and FR4_ $\left.Y_{b}\right)$, and the other two are green (FR4_ $Y_{a}$ and FR4_G $G_{b}$ ). The subscripts " $a$ " and " $b$ " stand for the samples provided by different manufacturers.

For clarity, the retrieved data are classified into two groups: the low-loss and the high-loss groups. The relative permittivity $\left(\varepsilon_{r} \equiv \operatorname{Re}\left[\varepsilon_{s}\right] / \varepsilon_{0}\right)$ of the low-loss group is plotted in Figure 4 . The loss tangent $\left(\tan \delta \equiv \operatorname{Im}\left[\varepsilon_{s}\right] / \operatorname{Re}\left[\varepsilon_{s}\right]\right)$ is not shown, because it is too low to test (less than $\left.10^{-3}\right)$ by the present scattering scheme. Instead, the conventional cavity methods with strong field enhancement are more suitable [12,13]. Among these low-loss polymers, PTFE exhibits the lowest $\varepsilon_{r}$ of around 2.14; PE and PP, belonging to the polyolefin, show very close $\varepsilon_{r}$ of around 2.45, and PVC and PC have relatively large $\varepsilon_{r}$, ranging from 2.8 to 2.9 . 


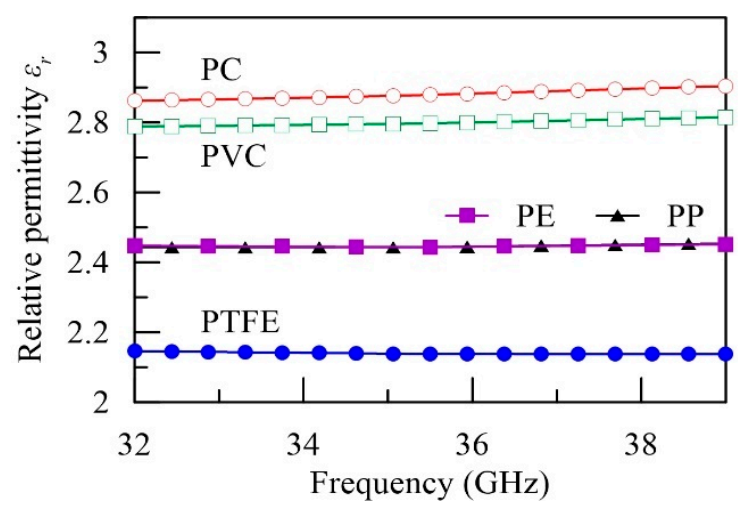

Figure 4. Relative permittivity of the low-loss materials.

Regarding the high-loss group, $\varepsilon_{r}(\tan \delta)$ is presented in Figure $5 \mathrm{a}, \mathrm{b}$. We found that PMMA and PVDF exhibit quite similar $\varepsilon_{r}$ around 2.77, while PVDF is relatively high-loss, manifested in its larger $\tan \delta$ around 0.02. On the other hand, the four FR4 boards possess higher and more diverse $\varepsilon_{r}$ (from 4.2-5.5). The higher dielectric constant and the stronger dielectric loss might result from their complex compositions, including epoxy matrices (typical $\left.\varepsilon_{r} \sim 3.5-4\right)$ [17], fiberglass $\left(\varepsilon_{r} \sim 3-15\right)$ for reinforcement [40], functional fillers, and dyeing materials. Although the concentrations of the latter two are usually low, they can still greatly alter the effective permittivity of the composite due to their high-K nature [41,42]. Besides, our data show that the FR4 boards provided by different manufacturers reveal very different permittivity even if the colors are the same (e.g., FR4_Y $Y_{a}$ vs. FR4_ $\left.Y_{b}\right)$. Such difference must result in very distinct circuit impedances at the high-frequency (5G) regime. This observation manifests the importance of our work, which can easily provide the accurate electrical properties of the targeted boards before any circuit designs. The data at $36 \mathrm{GHz}$ were extracted and summarized in Table 1 with error bars, showing fairly good agreement with the previously reported values (given in the parentheses) [26-36].
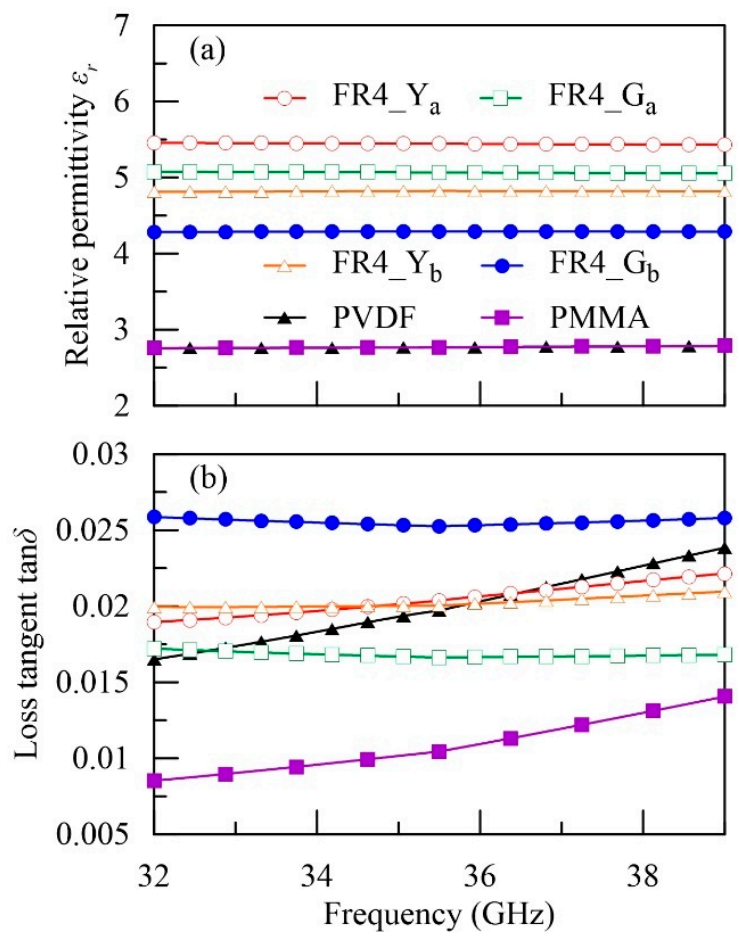

Figure 5. (a) Relative permittivity and (b) loss tangent of the high-loss materials. 
Table 1. The dielectric properties of the plastics at $36 \mathrm{GHz}$.

\begin{tabular}{|c|c|c|}
\hline Material & $\varepsilon_{r}$ & $\tan \delta$ \\
\hline PTFE & $\begin{array}{c}2.14 \pm 0.3 \% \\
(1.95)[26]\end{array}$ & $<0.001$ \\
\hline $\mathrm{PP}$ & $\begin{array}{c}2.45 \pm 0.1 \% \\
(2.29 \sim 2.30)[27]\end{array}$ & $<0.001$ \\
\hline PE & $\begin{array}{c}2.44 \pm 0.6 \% \\
(2.35 \sim 2.37)[27]\end{array}$ & $<0.001$ \\
\hline PVC & $\begin{array}{l}2.8 \pm 0.3 \% \\
(2.50)[28]\end{array}$ & $<0.005$ \\
\hline PC & $\begin{array}{c}2.88 \pm 0.1 \% \\
(2.76)[29]\end{array}$ & $<0.001$ \\
\hline PVDF & $\begin{array}{c}2.77 \pm 0.3 \% \\
(1.5 \sim 10)[30-33]\end{array}$ & $\begin{array}{c}0.02 \pm 11.8 \% \\
(0.02 \sim 0.16)[30]\end{array}$ \\
\hline PMMA & $\begin{array}{c}2.77 \pm 0.1 \% \\
(2.60 \sim 2.67)[27]\end{array}$ & $\begin{array}{c}0.011 \pm 16.9 \% \\
(0.015 \sim 0.061)[27]\end{array}$ \\
\hline FR4_G ${ }_{b}$ & $\begin{array}{c}4.29 \pm 0.3 \% \\
(3.5 \sim 5)[34-36]\end{array}$ & $\begin{array}{c}0.026 \pm 4.6 \% \\
(0.015 \sim 0.02)[18-20]\end{array}$ \\
\hline FR4_Y $Y_{b}$ & $4.82 \pm 0.2 \%$ & $0.02 \pm 3.1 \%$ \\
\hline FR4_G $\mathrm{G}_{\mathrm{a}}$ & $5.06 \pm 0.2 \%$ & $0.017 \pm 5.2 \%$ \\
\hline FR4_Y $Y_{a}$ & $5.44 \pm 0.8 \%$ & $0.02 \pm 7.3 \%$ \\
\hline
\end{tabular}

\section{Conclusions}

In Summary, this study established a broadband measurement system for fast dielectric characterization. By using the open-ended probe operating in the circular $\mathrm{TE}_{01}$ mode, the nearfield measurement of freestanding sheet dielectrics was accomplished. Since the experimental setup is compact, the sample preparation time and the system assembling time were significantly saved, as compared to the conventional cavity methods and the transmission/reflection methods in waveguide systems. The highly confined $\mathrm{TE}_{01}$ fringing field also eliminated the complicated calibrations for diffraction, leading to more accurate and stable results. More than 10 polymers (PTFE, PP, PE, PVC, PC, PVDF, PMMA, and FR4) were characterized from $30 \mathrm{GHz}$ to $40 \mathrm{GHz}$, showing various dielectric constants ranging from 2.1-5.5 and loss tangents ranging from 0.001-0.03. We believe this novel characterization method can benefit the development of the designs, fabrications, and manufacture quality controls of $5 \mathrm{G}$ high-frequency devices.

Supplementary Materials: The following are available online at http://www.mdpi.com/2073-4360/12/9/1891/s1.

Author Contributions: H.-Y.Y. and T.-H.C. conceived the experiment, D.-R.H. experimented, H.-Y.Y. provided theoretical model, H.-Y.Y. and D.-R.H. analyzed the results. All authors have read and agreed to the published version of the manuscript.

Funding: This work was funded by the Ministry of Science and Technology of Taiwan under grant No. MOST 107-2112-M-007-015-MY3.

Conflicts of Interest: The authors declare no conflict of interest.

\section{References}

1. Lie, D.Y.C.; Mayeda, J.C.; Li, Y.; Lopez, J. A review of 5G power amplifier design at cm-wave and mm-wave frequencies. Wirel. Commun. Mob. Comput. 2018, 2018, 6793814. [CrossRef]

2. Hu, S.; Wang, F.; Wang, H. A 28-/37-/39-GHz linear Doherty power amplifier in silicon for 5G applications. IEEE J. Solid-State Circuits 2019, 54, 1586-1599. [CrossRef]

3. Jilani, S.F.; Munoz, M.O.; Abbasi, Q.H.; Alomainy, A. Millimeter-wave liquid crystal polymer based conformal antenna array for 5G applications. IEEE Antennas Wirel. Propag. Lett. 2018, 18, 84-88. [CrossRef] 
4. Ali, M.; Liu, F.; Watanabe, A.; Raj, P.M.; Sundaram, V.; Tentzeris, M.M.; Tummala, R.R. First demonstration of compact, ultra-thin low-pass and bandpass filters for $5 \mathrm{~g}$ small-cell applications. IEEE Microw. Wirel. Compon. Lett. 2018, 28, 1110-1112. [CrossRef]

5. Su, C.S.; Chang, T.M. Manipulating the permittivities and permeabilities of epoxy/silver nanocomposites over a wide bandwidth. Appl. Phys. Lett. 2020, 116, 202904. [CrossRef]

6. Li, H.; Yang, J.; Dong, S.; Tian, F.; Li, X. Low dielectric constant polyimide obtained by four kinds of irradiation sources. Polymers 2020, 12, 879. [CrossRef]

7. Chen, M.; Zhou, W.; Zhang, J.; Chen, Q. Dielectric property and space charge behavior of polyimide/silicon nitride nanocomposite films. Polymers 2020, 12, 322. [CrossRef] [PubMed]

8. Kim, C.S.; Jo, S.J.; Lee, S.W.; Kim, W.J.; Baik, H.K.; Lee, S.J.; Hwang, D.K.; Im, S. High-k and low-k nanocomposite gate dielectrics for low voltage organic thin film transistors. Appl. Phys. Lett. 2006, 88, 243515. [CrossRef]

9. Chhajed, S.; Schubert, M.F.; Kim, J.K.; Schubert, E.F. Nanostructured multilayer graded-index antireflection coating for Si solar cells with broadband and omnidirectional characteristics. Appl. Phys. Lett. 2008, 93, 251108. [CrossRef]

10. Yao, H.Y.; Jiang, J.Y.; Cheng, Y.S.; Chen, Z.Y.; Her, T.-H.; Chang, T.-H. Modal analysis and efficient coupling of TE_01 mode in small-core THz Bragg fibers. Opt. Express 2015, 23, 27266-27281. [CrossRef]

11. Afsar, M.N.; Birch, J.R.; Clarke, R.N.; Chantry, G.W. The measurement of the properties of materials. Proc. IEEE 1986, 74, 183-199. [CrossRef]

12. Chao, H.-W.; Chang, T.-H. Wide-range permittivity measurement with a parametric-dependent cavity. IEEE Trans. Microw. Theory Tech. 2018, 66, 4641-4648. [CrossRef]

13. Chao, H.W.; Wong, W.S.; Chang, T.H. Characterizing the complex permittivity of high-k dielectrics using enhanced field method. Rev. Sci. Instrum. 2015, 86, 114701. [CrossRef] [PubMed]

14. Chiu, T. Dielectric constant measurement technique for a dielectric strip using a rectangular waveguide. IEEE Trans. Instrum. Meas. 2003, 52, 1501-1508. [CrossRef]

15. Cohn, S.; Kelly, K. Microwave Measurement of High-Dielectric- Constant Materials. IEEE Trans. Microw. Theory Tech. 1966, 14, 406-410. [CrossRef]

16. Taherian, M.; Yuen, D.; Habashy, T.; Kong, J. A coaxial-circular waveguide for dielectric measurement. IEEE Trans. Geosci. Remote Sens. 1991, 29, 321-330. [CrossRef]

17. Su, S.-C.; Yao, H.-Y.; Chang, T.-H. Characterization of ferrites using a fully loaded waveguide system. J. Magn. Magn. Mater. 2020, 505, 166712. [CrossRef]

18. Gagnon, N.; Shaker, J.; Berini, P.; Roy, L.; Petosa, A. Material characterization using a quasi-optical measurement system. IEEE Trans. Instrum. Meas. 2003, 52, 333-336. [CrossRef]

19. Gagnon, N.; Shaker, J.; Roy, L.; Petosa, A.; Berini, P. Low-cost free-space measurement of dielectric constant at Ka band. IEE Proc.-Microw. Antennas Propag. 2004, 151, 271-276. [CrossRef]

20. Skocik, P.; Neumann, P. Measurement of Complex Permittivity in Free Space. Procedia Eng. 2015, 100, $100-104$. [CrossRef]

21. Jeon, T.-I.; Grischkowsky, D. Characterization of optically dense, doped semiconductors by reflection $\mathrm{THz}$ time domain spectroscopy. Appl. Phys. Lett. 1998, 72, 3032-3034. [CrossRef]

22. Shen, Y.; Lo, T.; Taday, P.F.; Cole, B.E.; Tribe, W.R.; Kemp, M.C. Detection and identification of explosives using terahertz pulsed spectroscopic imaging. Appl. Phys. Lett. 2005, 86, 241116. [CrossRef]

23. Cunningham, P.D.; Valdes, N.N.; Vallejo, F.A.; Hayden, L.M.; Polishak, B.; Zhou, X.-H.; Luo, J.; Jen, A.K.; Williams, J.C.; Twieg, R.J. Broadband terahertz characterization of the refractive index and absorption of some important polymeric and organic electro-optic materials. J. Appl. Phys. 2011, 109, 043505. [CrossRef]

24. Yu, C.F.; Chang, T.H. High-performance circular TE/sub 01/-mode converter. IEEE Trans. Microw. Theory Tech. 2005, 53, 3794-3798.

25. Chang, T.-H.; Yu, B.R. High-power millimeter-wave rotary joint. Rev. Sci. Instrum. 2009, 80, 34701.

26. Abbas, Z.; Pollard, R.; Kelsall, R.W. Complex permittivity measurements at Ka-Band using rectangular dielectric waveguide. IEEE Trans. Instrum. Meas. 2001, 50, 1334-1342. [CrossRef]

27. Chang, T.; Zhang, X.; Zhang, X.; Cui, H.-L. Accurate determination of dielectric permittivity of polymers from $75 \mathrm{GHz}$ to $16 \mathrm{THz}$ using both S-parameters and transmission spectroscopy. Appl. Opt. 2017, 56, 3287-3292. [CrossRef] 
28. Murata, K.-I.; Hanawa, A.; Nozaki, R. Broadband complex permittivity measurement techniques of materials with thin configuration at microwave frequencies. J. Appl. Phys. 2005, 98, 084107. [CrossRef]

29. Riddle, B.; Baker-Jarvis, J.; Krupka, J. Complex permittivity measurements of common plastics over variable temperatures. IEEE Trans. Microw. Theory Tech. 2003, 51, 727-733. [CrossRef]

30. Zhang, X.-J.; Wang, G.-S.; Cao, W.-Q.; Wei, Y.-Z.; Cao, M.; Guo, L. Fabrication of multi-functional PVDF/RGO composites via a simple thermal reduction process and their enhanced electromagnetic wave absorption and dielectric properties. RSC Adv. 2014, 4, 19594-19601. [CrossRef]

31. Li, K.C.; Wang, H.; Xiang, F.; Liu, W.H.; Yang, H.B. Surface functionalized $\mathrm{Ba}_{0.6} \mathrm{Sr}_{0.4} \mathrm{TiO}_{3} /$ poly (vinylidene fluoride) nanocomposites with significantly enhanced dielectric properties. Appl. Phys. Lett. 2009, 95, 202904. [CrossRef]

32. Thomas, P.; Varughese, K.T.; Dwarakanath, K.; Varma, K.B.R. Dielectric properties of Poly(vinylidene fluoride) $/ \mathrm{CaCu}_{3} \mathrm{Ti}_{4} \mathrm{O}_{12}$ composites. Compos. Sci. Technol. 2010, 70, 539-545. [CrossRef]

33. Huang, E.-Q.; Zhao, J.; Zha, J.-W.; Zhang, L.; Liao, R.-J.; Dang, Z.-M. Preparation and wide-frequency dielectric properties of $\left(\mathrm{Ba}_{0.5} \mathrm{Sr}_{0.4} \mathrm{Ca}_{0.1}\right) \mathrm{TiO}_{3} /$ poly(vinylidene fluoride) composites. J. Appl. Phys. 2014, 115, 194102. [CrossRef]

34. Djordjevic, A.; Biljie, R.; Likar-Smiljanic, V.; Sarkar, T. Wideband frequency-domain characterization of FR-4 and time-domain causality. IEEE Trans. Electromagn. Compat. 2001, 43, 662-667. [CrossRef]

35. Chang, S.-H.; Kuan, H.; Wu, H.-W.; Yang, R.-Y.; Weng, M.-H. Determination of microwave dielectric constant by two microstrip line method combined with EM simulation. Microw. Opt. Technol. Lett. 2006, 48, $2199-2201$. [CrossRef]

36. Yamacli, S.; Ozdemir, C.; Akdağli, A. A Method for Determining the Dielectric Constant of Microwave PCB Substrates. Int. J. Infrared Millim. Waves 2007, 29, 207-216. [CrossRef]

37. Pozar, D.M. Microwave Engineering; John Wiley \& Sons: Hoboken, NJ, USA, 2009.

38. Chen, N.C.; Yu, C.F.; Yuan, C.P.; Chang, T.-H. A mode-selective circuit for TE01 gyrotron backward-wave oscillator with wide-tuning range. Appl. Phys. Lett. 2009, 94, 101501. [CrossRef]

39. Yao, H.-Y.; Chang, T.-H. Effect of High-Order Modes on Tunneling Characteristics. Prog. Electromagn. Res. 2010, 101, 291-306. [CrossRef]

40. Wallenberger, F.T.; Bingham, P.A. Fiberglass and Glass Technology: Energy-Friendly Compositions and Applications; Springer: New York, NY, USA, 2010.

41. Cheng, K.-C.; Lin, C.-M.; Wang, S.-F.; Lin, S.-T.; Yang, C.-F. Dielectric properties of epoxy resin-barium titanate composites at high frequency. Mater. Lett. 2007, 61, 757-760. [CrossRef]

42. Wu, C.-C.; Yang, C.-F. Investigating the mechanical properties of high dielectric constant polyetherimide/ $\left(\mathrm{Ba}_{0.8} \mathrm{Sr}_{0.2}\right)\left(\mathrm{Ti}_{0.9} \mathrm{Zr}_{0.1}\right) \mathrm{O}_{3}$ composites. Compos. Part B Eng. 2011, 42, 1799-1802. [CrossRef] 